\title{
Laudation for Academician Professor Dr. Boris F. Myasoedov
}

\author{
A. Chatt
}

(C) Akadémiai Kiadó, Budapest, Hungary 2012

Academician Professor Dr. Boris F. Myasoedov was born in Russia. His scientific career started with the diploma work at the Vernadsky Institute of Geochemistry and Analytical Chemistry, USSR Academy of Sciences. In 1953 he graduated from Mendeleev Moscow Chemical Technological University. In 1964 he received his Candidate of Sciences (equivalent to $\mathrm{PhD}$ ) on the thesis entitled "Development of methods for separation and determination of protactinium". The monograph "Analytical chemistry of protactinium" was awarded the Khlopin Prize by the USSR Academy of Sciences in the field of radiochemistry in 1975.

During 1954-1960, Dr. Myasoedov worked on the synthesis, isolation and study of chemical properties of $\mathrm{Md}$, No, Lr and element 104 (now Rf) in different solutions in Moscow and Dubna under the guidance of Professors I. V. Kurchatov and G. N. Flerov. Later on he became the Head of the group on the synthesis and properties of volatile compounds of beta-diketones of $\mathrm{Am}, \mathrm{Cm}, \mathrm{Bk}, \mathrm{Cf}$

Prepared by Professor Dr. A. Chatt (Halifax, Canada), Chairman of the Hevesy Medal Award Selection Panel 2012 and President of the International Committee on Activation Analysis (ICAA).

Narrated by Professor Dr. A. Chatt on behalf of Dr. Stephen LaMont (General Chair) and Dr. Samuel Glover (Technical Program Chair) of the Ninth International Conference on Methods and Applications of Radioanalytical Chemistry (MARC-IX) held at Sheraton Keauhou Bay Hotel, Kailua-Kona, HI, U.S.A., during 2012 March 25-30. Hevesy Medal and Scroll were presented at MARC-IX by Professor Dr. A. Chatt on behalf of Professor Dr. Dr. h.c. Tibor Braun (Budapest, Hungary), Editor-in-Chief, Journal of Radioanalytical and Nuclear Chemistry.

\section{A. Chatt $(\square)$}

Department of Chemistry, Trace Analysis Research Centre,

Dalhousie University, 6274 Coburg Road, Room 212,

P.O. Box 15000, Halifax, NS B3H 4R2, Canada

e-mail: a.chatt@dal.ca and Es using gas chromatography. In 1960 he became the project leader on chemical studies of Pa related to problem of homogeneous thorium reactor. He went to the Radium Institute in Paris for training under Professor M. Haissinsky, and their collaboration continued until 1972.

Since 1969 Dr. Myasoedov has been the Head of basic research on chemistry of trans-uranium elements (TUE). The research topics include: investigations of chemical properties and stabilization of TUE in unusual oxidation states. He was awarded Doctor of Sciences (DSc) in 1976 for the "Study of the properties of trans-plutonium elements in different redox states: Novel separation and determination methods". In 1977 he became the Professor of analytical chemistry. In 1986 Professor Myasoedov was awarded the prestigious State Prize of the USSR for achievements in "Chemical Behavior of the Metals in Unusual Oxidation States".

Professor Myasoedov headed many projects including bidentated neutral organophosphorus compounds (BNOC) for the extraction of actinides from acidic solutions leading to the discovery of "aryl effect" and so called "Russian technological scheme" of extraction of trans-plutonium and rare earth elements using BNOC. This scheme has been successfully tested in the nuclear fuel reprocessing plant "Mayak". Around the same time he investigated the synthesis and properties of zirconium phosphate for the separation of Am, Cm, Cf and Bk.

In recent years, Professor Myasoedov has been interested in the development of low-waste and energy-saving methods for spent nuclear fuel reprocessing as well as for the development of new technique for immobilizing the radionuclides in mineral-like matrices. He has been working for the last 30 years or so in radioecology. For monitoring remediation of radionuclide-contaminated territories in the vicinity of nuclear fuel cycle enterprises, Professor Myasoedov and 
coworkers developed ingenious procedures for preconcentration, separation, and determination of actinides and other radionuclides in bottom sediments, soils, and underground waters. It was found for the first time that, in the regions of the Mayak Production Association and Siberian Chemical Plant, Joint-Stock Company, long-lived radionuclides migrated in the form of colloidal particles. This discovery allowed development of efficient measures against migration and filtration of radionuclides.

Professor Myasoedov and coworkers patented a process for the production of radiopharmaceuticals based on highly purified ${ }^{225} \mathrm{Ac}$ for alpha-particle therapy of tumors. Professor Myasoedov has been the Head of the Laboratory of radiochemistry at Vernadsky Institute for more than 30 years. This laboratory is recognized as one of the leading centers in radiochemistry, analytical chemistry of radioactive elements, and radioecology. In 1994 Professor Myasoedov has been elected as a full member of the Russian Academy of Sciences.

The scientific achievements of Professor Myasoedov and his colleagues have been reflected in more than 750 papers in Russian and international journals, seven monographs, and 25 patents and inventor's certificates. Six DSc and $31 \mathrm{PhD}$ dissertations have been defended under his guidance. He heads the leading scientific school "Actinide Elements: Properties, Behavior in Technogenic and Natural Processes," which is funded by the Russian Federation President's grant.

Professor Myasoedov successfully combines creative scientific activity with professional organizational work. Now he is the Editor-in-Chief of "Russian Radiochemistry", member of the editorial board of Russian "Journal of
Analytical Chemistry", "Mendeleev's Communications", and member of editorial boards of several international journals, such as Solvent Extraction and Ion Exchange, J. Radioanal. Nucl. Chem, Problems of Analytical Chemistry etc., Chair of the Interdepartmental Scientific Council on Radiochemistry at the Russian Academy of Sciences and the "Rosatom" State Atomic Energy Corporation of the Russian Federation, Vice-President of the National Committee of Russian Chemists among numerous other duties.

Academician Professor Dr. Boris F. Myasoedov is a distinguished scientist, an acknowledged scientific leader in the fields of radiochemistry, analytical chemistry of radioactive elements, and radioecology. It is indeed a pleasure to honor him with the Hevesy Medal Award 2012.

Hevesy Medal Award 2012 presentation photo: courtesy of Dr. Vladimir Kolotov. From left to right: Dr. Stephen LaMont, Professor Dr. Boris F. Myasoedov, Professor Dr. A. Chatt, and Dr. Samuel Glover.

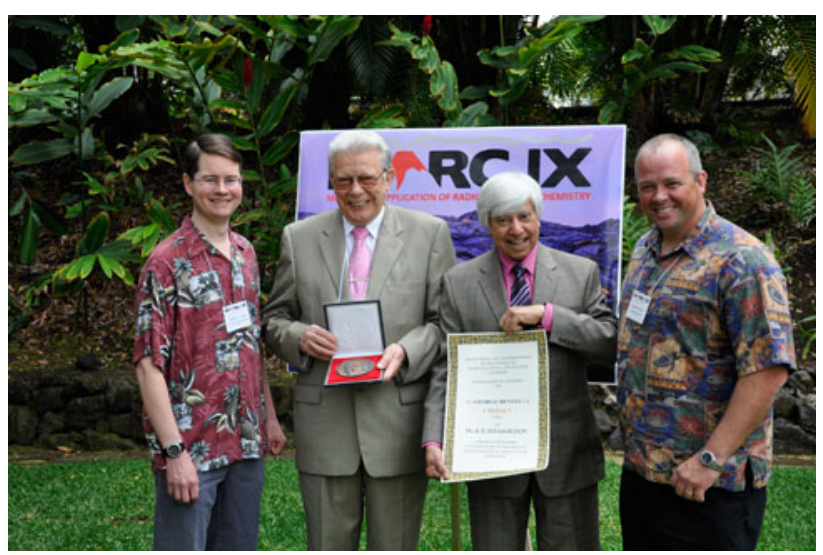

